\title{
Soft control of swarms - analytical approach
}

\author{
Guillaume Sartoretti* and Max-Olivier Hongler \\ $\mathrm{EPFL} / \mathrm{STI} / \mathrm{IMT} / \mathrm{LPM}$
}

June 4, 2013

\begin{abstract}
We analytically study the collective dynamics of mutually interacting heterogeneous agents evolving in a random environment. Our formal framework consist of a collection of $N$ scalar drifted Brownian motions (BM) diffusing on $\mathbb{R}$. The mutual interactions are introduced via a ranked-based, real-time mechanism always endowing the laggard, (i.e the agent with the leftmost position), with an extra positive drift. The extra drift generates a net tendency for of any agents not to remain the laggard of the society. For well chosen individual and extra laggard's drifts, the agents organize with time to flock towards a tight and stable traveling spatial pattern. For a population of $(N-1)$ identical agents and an atypic fellow, (called hereafter the shill), we are able to analytically discuss the dynamics. In particular we exhibit how a single turbulent shill, stylized here by a ballistic diffusion process, can destroy the cohesion of a swarm. Conversely, we also analytically show how a single shill, via interactions with its fellows, is able to safely pilot a whole swarm to avoid an obstacle. A series of simulations experiments comfort our analytic findings.
\end{abstract}

\section{Homogeneous versus heterogeneous mutually in- teracting stochastic agents}

The capability of a collection of interacting stochastic agents to exhibit an emergent collective behaviour (i.e flocking behaviour) even in random environments stimulates a strong research activity devoted to both experimental and theoretical modeling approaches. For suitable range of mutual interactions, flocking (phase) transitions, namely the self-organized capability to create finite and persistent spatio-temporal patterns, are observed (see $[1,3-5,10,13,14]$ ).

Agents societies can be composed of either dynamically homogeneous or heterogeneous individuals requiring for each case drastically different approaches. For large and homogeneous population of agents, the classical statistical mechanics concepts and in particular, the mean-field description (MF) directly offers an appropriate tool to analytically discuss the global dynamics. In the MF description, one

\footnotetext{
*Supported by the Swiss National Funds for Scientific Research
} 
basically assumes that the behaviour of the global society can be characterized by the dynamics of a single representative agent which feels its fellows' interactions via an effective external field. In most circumstances however, homogeneity fails, and therefore a growing attention is now paid to heterogeneous populations. Heterogeneity occurs typically when one or several masked agents, that we shall from now on refer to as shills, exhibit leaders or troublemakers behaviours. These shills are not recognized by the regular agents which see them as ordinary fellows. Hence the interaction rules between ordinary fellows and shills remain unchanged. The presence of shills can strongly alter the ultimate evolution of the whole society and it is the central goal here to analytically study this problematic. Our approach involves assemblies of interacting stochastic agents in which a single shill exhibits a different individual behaviour. All agents, including the shill, interact with their fellows with fixed given rules. The paradigmatic vision of this situations has been currently explored in ethology where one fake individual is introduced among schools of fishes, cockroaches, or newborn chicken, etc. The shill is able to ultimately pilot the whole population $([2,7,11])$. This basic mechanism also referred as the soft control of a population $([6,15-17])$ is the core of our present paper. As heterogeneous societies preclude MF approaches, rather few analytical results for heterogeneous cases are yet available. Recent mathematical models of heterogeneous rank-based interacting Brownian motions (RBM), introduced in finance $([1,4,10,13])$, will be used in the sequel to analytically approach the soft control problematic. We shall analytically show how a troublemaker can break the cohesion of an initially tight swarm and how a single agent can efficiently incitate its fellows to achieve a preassigned task.

Our paper is organized as follows: we first recall in section 2 relevant properties of RBM, also known in economy as the Atlas models. In section 3, we use the RBM's framework to show how a troublemaker is able to smash an initially tight swarm. Our individual dynamics are one-dimensional diffusion processes with piecewise constant drifts except for the shill, which will be assumed to be driven by a ballistic process with quadratic variance in time. The ballistic noise is itself generated by a simple non-Gaussian diffusive stochastic process with nonlinear drift. In section 4, we address the dual soft control problem in which a shill is used to steer the whole collection of RBM's towards slots that are drilled trough an obstacle board.

\section{Rank-based Brownian motions - Atlas model}

Our approach makes extensive use of recent results derived in [13], that we now briefly summarize. Let us consider a collection of $N$ interacting agents diffusing according to the class of processes:

$$
\mathrm{d} Y_{i}(t)=\left(\sum_{k=1}^{N} g_{k} 1_{Q_{k}(i)}\{Y(t)\}+\gamma_{i}+\gamma\right) \mathrm{d} t+\sigma_{i} \mathrm{~d} W_{i}(t), \quad Y_{i}(0)=y_{i}, t \in \mathbb{R}^{+},
$$

where $Y(t)=\left(Y_{1}, Y_{2}, \cdots, Y_{N}\right) \in \mathbb{R}^{N}$ and $\mathrm{d} W_{i}(t)$ are $N$ independent standard White Gaussian Noise processes (WGN) processes. The indicator function $1_{Q_{k}}$ in Eq.(1) effectively generates mutual interactions. The effective, time-dependent drift 
component $g_{k}$ entering into the drifts $\left(g_{k}+\gamma_{i}+\gamma\right)$ of the $N$ Brownian motions on $\mathbb{R}$, is rank-based. Namely, it is instantaneously adjusted according to the position (i.e. the rank) occupied by each agent with respect to the remaining $(N-1)$ fellows. The constant drift components $\gamma_{i}$ are name-based, i.e. they are definitely assigned to each individual agent ( $\gamma_{i}$ is time-independent). Finally, a constant drift component $\gamma$ can be added, which is common to all agents. Accordingly Eq.(1) describes a collection of $N$ diffusion processes having piecewise deterministic drifts. The somehow simpler situation obtained when $\gamma_{i} \equiv 0 \forall i$ has been thoroughly studied in [10].

In the sequel and without loss of generality, we systematically choose $\gamma$ to be the (average) barycentric speed of the swarm. This is achieved, provided one has:

$$
\sum_{k=1}^{N}\left[g_{k}+\gamma_{k}\right]=0
$$

For future use, we introduce the following notations and definitions:

1. We write

$$
\bar{Y}(t)=\frac{1}{N} \sum_{k=1}^{N} Y_{k}(t), \quad \bar{Y} \in \mathbb{R},
$$

for the barycenter position. The set $\Sigma_{N}$ stands for the set of all the permutations of $\{1,2, \ldots, N\}$. It is proven in [13] that, for almost every initial conditions and when the set of constraints

$$
\sum_{k=1}^{l}\left[g_{k}+\gamma_{p(l)}\right]<0
$$

are fulfilled for all possible permutations $p=(p(1), \ldots, p(N)) \in \Sigma_{N}$, then the $N$ deviations processes:

$$
\tilde{Y}_{i}(t)=\left[Y_{i}(t)-\bar{Y}(t)\right]
$$

converge to stationary probability measures. Note that Eq.(2) yields therefore a set of $(N-1) \times\left|\Sigma_{N}\right|=N !(N-1)$ constraints to be verified.

2. When all constraints in Eq.(2) are fulfilled, the dynamics given by Eq.(1) then converges to a tight swarm described by a stationary multi-variate process characterizing the $(N-1)$-gap processes $\Xi_{i}(t)$

$$
\Xi_{i}(t)=\left[Y_{i+1}(t)-Y_{i}(t)\right]
$$

The associated probability density $\psi(z), z \in \mathbb{R}_{+}^{N-1}$ can be written as a sumof-product-of-exponential form:

$$
\psi(z)=\left(\sum_{q \in \Sigma_{N}} \prod_{k=1}^{N-1} \lambda_{p, k}^{-1}\right)^{-1} \sum_{p \in \Sigma_{N}} \exp \left(-\left\langle\lambda_{p}, z\right\rangle\right) \quad\left(\lambda_{p}=\left(\lambda_{p, 1} \lambda_{p, 2} \ldots \lambda_{p, N-1}\right)\right)
$$


with the parameters explicitly given by

$$
\lambda_{p, k}=\frac{-4 \sum_{l=1}^{k}\left(g_{l}+\gamma_{p(l)}\right)}{\sigma_{k}^{2}+\sigma_{k+1}^{2}}, \quad p \in \Sigma_{N}, k \in\{1, \ldots, N-1\}
$$

Remark. It is worth observing that the diffusion coefficients $\sigma_{i}$ do not enter into the set of tightness constraints given in Eq.(2). However, the $\sigma_{i}$ 's do enter into the parameters Eq.(4) characterizing the stationary probability measure.

\section{How does a super-diffusive fellow smash tightness}

We first investigate how a single "turbulent" fellow can destroy the cohesion of a tight swarm. To this aim, we use the RBM formalism introduced in section (2). We consider a configuration involving $(N-1)$ identical mutually interacting RBM's, referred from now on as the regular agents, interacting with a single fellow, the shill, say agent number one, which is itself driven by a ballistic diffusion process, (remember from Eq.(1) that the $(N-1)$ regular agents are driven by independent WGN's). The ballistic process, to be introduced below, exhibits a variance $\propto t+\beta t^{2}$ with a ballistic parameter $\beta \geq 0$ a constant. For $\beta=0$, the shill simply behaves as a regular fellow and therefore, in this $\beta=0$ limit, our global dynamics reduces to a standard version of Eq.(1). We set the specific parameters: $g_{N}=(N-1) g$ and $g_{k}=-g$ for $1 \leq k \leq N-1$. In addition, we shall further assume that:

$$
\begin{aligned}
& \text { i) } \quad \sum_{i=1}^{N} \gamma_{i}=0, \\
& \text { ii) } \quad \max _{1 \leq i \leq N} \gamma_{i}<g .
\end{aligned}
$$

The couple of constraints i) and ii) imply that the Eq.(2) is fulfilled. Indeed, we can directly verify that :

$$
-g+\gamma_{p(1)}<0, \quad-2 g+\gamma_{p(1)}+\gamma_{p(2)}<0, \quad \cdots, \quad-(N-1) g+\sum_{k=1}^{N-1} \gamma_{p(k)}<0 .
$$

Hence, for $\beta=0$, our collection of agents behaves as a tight swarm enjoying a stationary joint probability law for the inter-distance between successive agents.

Let us now focus on $\beta>0$. Now, the shill $Y_{1}(t)$ behaves as a turbulent fellow which interact with the remaining $(N-1)$ regular fellows. Specifically the dynamics of $Y_{1}(t)$ is chosen to be described by:

$$
\mathrm{d} Y_{1}(t)=\left(\sum_{k=1}^{N} g_{k} 1_{Q_{k}(i)}\{Y(t)\}+\gamma_{1}+\gamma\right) \mathrm{d} t+\mathrm{d} Z(t), \quad Y_{1}(0)=y_{1} \quad t \in \mathbb{R}^{+},
$$

where the process $\mathrm{d} Z(t)$ is a super-diffusive ballistic noise generator introduced in $[8,9]$ :

$$
\mathrm{d} Z(t)=\{\beta \tanh [\beta Z(t)]\} \mathrm{d} t+\mathrm{d} W(t), \quad Z(t=0)=0 .
$$


The non-Gaussian Markov diffusion process $Z(t)$ given in Eq.(6) is fully characterized by its transition probability density $P(z, t \mid 0)$ which very simply reads:

$$
P(z, t \mid 0)=\frac{P_{+}(z, t \mid 0)+P_{-}(z, t \mid 0)}{2}, \quad P_{ \pm}(z, t \mid 0)=\frac{e^{-\frac{\beta^{2}}{2} t}}{\sqrt{2 \pi t}} e^{\frac{(x \pm \beta t)^{2}}{2 t}}
$$

with average $\langle Z(t)\rangle=0$ and variance

$$
\left\langle Z^{2}(t)\right\rangle=\int_{\mathbb{R}} z^{2} P(z, t \mid 0) d z=t+\beta^{2} t^{2} .
$$

The simple form given in Eq.(7) suggests the existence of an alternative representation for the ballistic noise $Z(t)$. Indeed, writing $B M_{ \pm \beta}(t)$ for the Brownian motions with $\pm \beta$ constant drifts, we observe that the transition probability for the process $Z(t)$ can be rewritten as $Z(t)=\mathcal{B} B M_{ \pm \beta}(t)$ where $\mathcal{B}$ is a symmetric Bernoulli r.v. taking the values \pm 1 with equal probability $1 / 2$. Hence, one realisation of the $Z(t)$ consists first in choosing, with probability $1 / 2$, one among the couple processes $B M_{ \pm \beta}(t)$ and then follow the realisation of the selected process, (see example 2 in $[12]$ and $[8,9])$.

We now come back to the dynamics jointly involving a turbulent fellow given by Eq. (5) and $(N-1)$ regular agents described by Eq.(1). We then view the $(N-1)$ regular agents as being infiltrated by a the shill $Y_{1}(t)$.

Now we have to investigate the values of $\beta$ enabling the swarm to remain tight, (i.e. does it exists a stationary probability measure for the intervals between successive agents). In view of the representation given in Eq.(8), for each realisation of the noise source $Z(t)$, we effectively deal with a standard RBM model with a re-normalized name-based drift of $Y_{1}(t)$, namely $\gamma_{1} \mapsto \gamma_{1} \pm \beta$ depending on the outcome of $\mathcal{B}$. Accordingly, to infer on the tightness of the swarm, we simply have to separately examine Eq.(2) for the couple of outcomes $\pm \beta$.

Realisation $+\beta$. Let us define $\gamma=\frac{\beta}{N}, \gamma_{1}=\frac{N-1}{N} \beta$ and $\gamma_{i}=-\gamma=-\frac{\beta}{N}$ for $2 \leq i \leq N$. The constraints Eq.(3) are required for the swarm to be tight. This yields, for $l=1$ and $p=(1,2,3 \ldots, N)$, to the most critical constraint:

$$
g_{1}+\gamma_{p(1)}<0 \Rightarrow-g+\frac{N-1}{N} \beta<0 \quad \Leftrightarrow \quad \beta<\frac{N}{N-1} g .
$$

Provided that Eq.(9) holds, the swarm remains tight when the $Z_{t}$ noise induces $\mathrm{a}+\beta$ extra drift.

Realisation $-\beta$. The same reasonning applied to the $-\beta$ case yields:

$$
\gamma=-\frac{\beta}{N}, \quad \gamma_{1}=-\frac{N-1}{N} \beta, \quad \gamma_{i}=\frac{\beta}{N} \quad(2 \leq i \leq N) .
$$

The critical constraint arises when $l=1$ and $\gamma_{p}:=\left(\frac{\beta}{N}, \ldots, \frac{\beta}{N},-\frac{N-1}{N} \beta\right)$ with:

$$
-g+\frac{\beta}{N}<0 \quad \Leftrightarrow \quad \beta<N \cdot g
$$


This implies that for $\beta<N \cdot g$, the swarm remains tight when the $Z_{t}$ noise induces a $-\beta$ extra drift.

The previous considerations can be summarized by observing that when the constraints Eq.(9) are fulfilled, so are also those given in Eq.(10). This suggests to distinguish an alternative "semi"-tight regime which arises when the constraints Eq.(10) alone are fulfilled. In this "semi"-tight case, the swarm exhibits a tight configuration when the $-\beta$ realisation is achieved and is not tight for the other alternative $+\beta$. This intrinsic asymmetry can be easily understood as our RBM dynamics only pushes the laggard towards the others. Hence, regarding the tightness, the shill is obviously less influential when the $-\beta$ extra drift is realised.

\subsection{Spatial dispersion of the agents}

The extra $\pm \beta$-drifts due to the super-diffusive process driving the shill dynamics obviously affect the spatial dispersion of the swarm. As all noise sources, (including the $d Z(t)$ process), have zero average, the barycenter of the whole population will remain unchanged by the presence of $d Z(t)$. However, due to the presence of the shill, an initial single-modal cluster of agents will, as time increases, be be split into two separately evolving population subgroups. Indeed, according to the $\pm \beta$ realisation taken by $d Z(t)$, the overall effect of the shill will either to steer the swarm towards negative or towards the positive directions; this generates the formation of two distinct clusters. Each cluster has an individual barycentric velocity: $\gamma=\frac{\gamma_{1}}{N}=$ $\frac{ \pm \beta}{N}$; this guarantees that the average barycenter remains unchanged. Specifically, whenever the shill's drift assumes the value $-\beta$, the shill has an overall propensity to stay at the rear of the swarm. Accordingly, all regular agents will, with high probability, be endowed with the rank-based drift $-g$. The regular agents hence possess a net tendency to be driven toward the negative direction on $\mathbb{R}$. Conversely, in presence of the $+\beta$ realisation, the shill is very likely to belong to the group of leaders. This impose to the remaining $(N-1)$ regular fellows to equally share, with alternations, the rank-based drift of $(N-1) g$ which drives the laggard. Therefore, with the $+\beta$ realisation, the whole population is very likely be driven towards the positive direction of $\mathbb{R}$. We now can isolate three regimes depending on the strength $\beta$.

a) $\beta<\beta_{c}:=\frac{N}{N-1} g$. In this regime, the shill is able to steer the whole population in one of the two possible directions and it itself remained attached to the swarm. In other words, a stationary probability measure exists for the distance between the agents, (i.e. all agents evolve in a single flock). Note however that the presence of the shill breaks an initially uni-modal spatial repartition into a bi-modal repartition.

For three agents, (i.e. one shill and two regular fellows), the resulting spatial repartition, obtained by simulation, is shown in Figure (1). In Figures (1), (2) and (3), the shill's position is represented in black, the others being displayed in grey. 


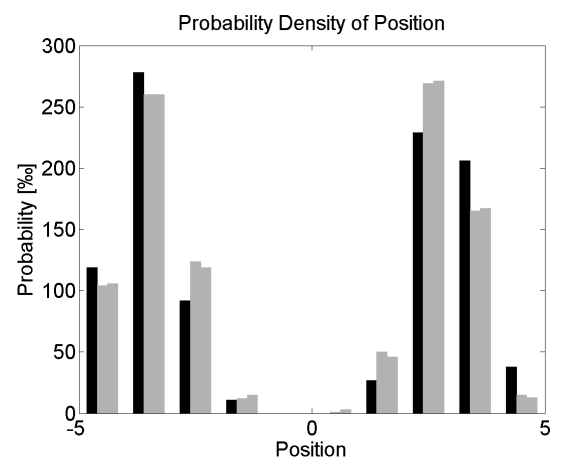

Figure 1: Probability of spatial repartition of the agents at $t_{\text {end }}=10$, numerical computation over $10^{3}$ runs, with $N=3, g=1, \beta=1.1<\beta_{c}=\frac{3}{2}$.

b) $\beta_{c} \leq \beta<g \cdot N$. Here the strength of the ballistic noise precludes to reach a global stationary state for the inter-distance processes between the $N$ agents. Indeed, the remormalized drift associated with the $+\beta$ realisation of $d Z(t)$, (i.e. $\hat{\gamma}_{1}=\gamma_{1}+\beta$ ), violates the constraint Eq.(9). However, for the $-\beta$ realisation, the constraint for $\hat{\gamma}_{1}=\gamma_{1}-\beta$ is fulfilled, implying that the shill remains flocked with its $(N-1)$ remaining fellows. An experiment with $\beta$ chosen in this range, shows the spatial repartition in Figure (2).
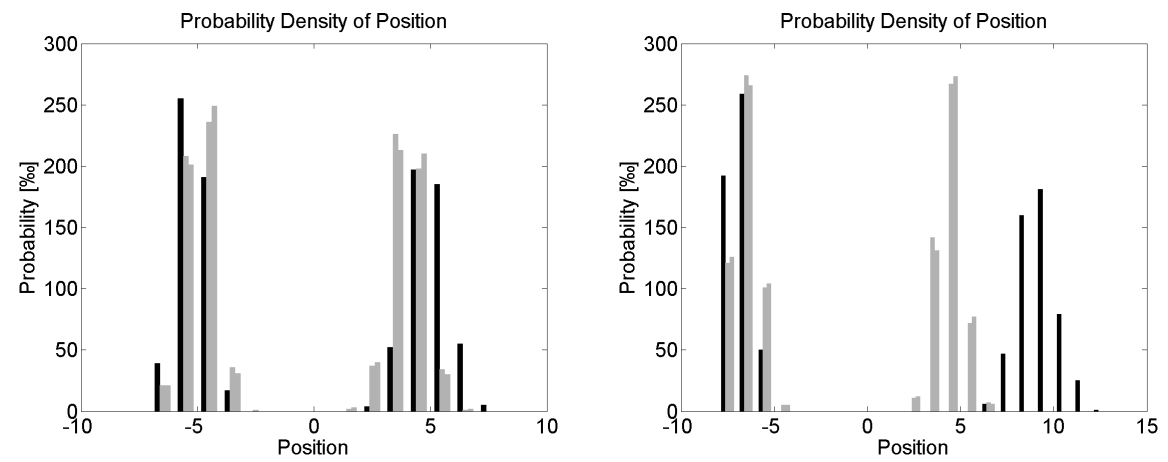

Figure 2: Left: End position distribution at $t_{\text {end }}=10$, numerical computation over $10^{3}$ runs, with $N=3, g=1, \beta=\beta_{c}=1.5$.

Right: End position distribution at $t_{\text {end }}=10$, numerical computation over $10^{3}$ runs, with $N=3, g=1, \beta=2>\beta_{c}$.

c) Finally, for $\beta>g \cdot N$, the shill becomes highly turbulent and the tightness constraints are never fulfilled. The shill escapes from the flock and the resulting spatial repartition is typically shown in Figure (3).

For all choices of the ballistic strength $\beta$, the numerical results intimately match the analytical predictions. In particular, the shill escapes from the flock when the critical ballistic strength $\beta=\beta_{c}$ is reached (for the $+\beta$ realisation, or when $\beta=N \cdot g$ 

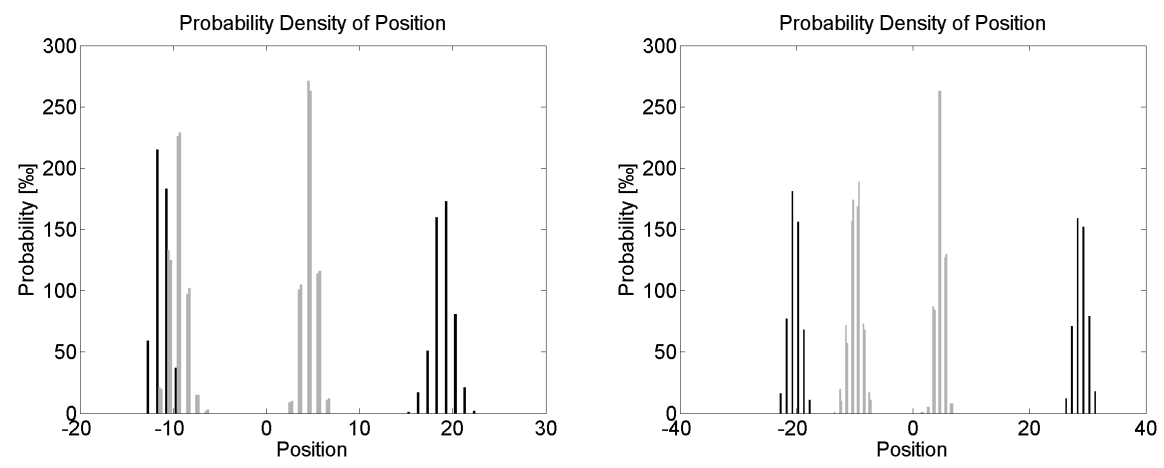

Figure 3: Left panel: Probability density of the position at $t_{\text {end }}=10$ for $N=3$, $g=1, \beta=N \cdot g=3$. The numerical computation includes $10^{3}$ runs,

Right panel: Probability density of the position at $t_{\text {end }}=10$ for $N=3, g=1$, $\beta=4>N \cdot g$. The numerical computation includes $10^{3}$ runs.

for the $-\beta$ realisation of $d Z(t)$. In these non-stationary regimes, the shill quits the $(N-1)$ - regular tight swarm with velocity $\mathcal{V}(\beta) \propto \beta$.

\subsection{Distance between successive agents}

Complementary to the tightness constraints, let us now briefly discuss the stationary probability measure which characterize the distances between successive agents. To this aim and for simplicity, we shall keep here identical diffusion coefficients, $\sigma_{i}=$ $\sigma=1(\forall i \in\{1,2, \ldots, N\})$, and choose $\beta$ in the range which ensures the existence of a global stationary regime, (i.e. ensures tightness), namely:

$$
0 \leq \beta<\beta_{c}=\frac{N}{N-1} g .
$$

Note however that, using, the results derived in [13], heterogeneous diffusion constants can also be analytically discussed. The distances separating consecutive agents defined in Eq.(3) can be explicitly calculated by using the results summarized in section (2). For agent $Y_{1}(t)$ and in view of the extra $\beta$-drift induced by the shill ballistic driving, we are now led to define a couple of name-based drifts vectors as:

$$
\gamma^{+}=\left(\begin{array}{llll}
\frac{N-1}{N} \beta & -\frac{\beta}{N} & \cdots & -\frac{\beta}{N}
\end{array}\right) \quad \text { and } \quad \gamma^{-}=\left(\begin{array}{cccc}
-\frac{N-1}{N} \beta & \frac{\beta}{N} & \cdots & \frac{\beta}{N}
\end{array}\right) .
$$

The coefficients entering into the inter-distances probability measure read

$$
\lambda_{p, k}^{ \pm}=-2 \sum_{l=1}^{k}\left[g_{l}+\gamma_{p(l)}^{ \pm}\right], \quad \forall p \in \Sigma_{N}, \quad 1 \leq k \leq N-1
$$

The fulfillment of the tightness constraints given in Eq.(2) ensures $\lambda_{p, k}^{ \pm}>0(\forall p, k)$. Hence, it results a couple of stationary probability densities, (one for each realisation $+\beta$ and $-\beta$ ), characterizing the inter-distance process $\Xi(t)$ (see Eq.(3)). One can explicitly write:

$$
\operatorname{Prob}\{z \leq \Xi(t) \leq z+d z \mid \pm \beta \text { is realised }\}=
$$




$$
=\mathcal{P}_{ \pm \beta}(z) d z=\left(\sum_{p \in \Sigma_{N}}\left(\prod_{k=1}^{N-1} \lambda_{p, k}^{ \pm}\right)^{-1}\right)^{-1} \sum_{p \in \Sigma_{N}} \exp \left(-\left\langle\lambda_{p}^{ \pm}, z\right\rangle\right) d z .
$$

The complete stationary probability density then reads:

$$
\mathcal{P}(z)=\frac{1}{2}\left[\mathcal{P}_{+\beta}(z)+\mathcal{P}_{-\beta}(z)\right] .
$$

This last expression consists in a rather complex form involving sum of products of exponentials, (as also does $\mathcal{P}_{ \pm \beta}(z)$ ). For the distance between agents, it is more informative to separately focus on the marginal densities obtained for each realisations $\pm \beta$ induced by $d Z(t)$. Let us now experimentally illustrate this situation.

\subsubsection{Illustration involving four agents $(N=4$}

The average distance between the shill and the first regular agent and the averages of the two distances between the three remaining regular agents can be explicitely computed for $N=4$. We obtain:

$$
\mathbb{E}_{ \pm \beta}[z]=\int_{\mathbb{R}_{+}^{3}} z \cdot \mathcal{P}_{ \pm \beta}(z) \mathrm{d} z=\Lambda^{ \pm} \int_{\mathbb{R}_{+}^{3}}\left(\sum_{p \in \Sigma_{4}} \exp \left(-\left\langle\lambda_{p}^{ \pm}, z\right\rangle\right)\right) \mathrm{d} z
$$

For $g=1$, and $0 \leq \beta<N g=4$, these inter-agents distances as a function of time are shown in Figure (4).
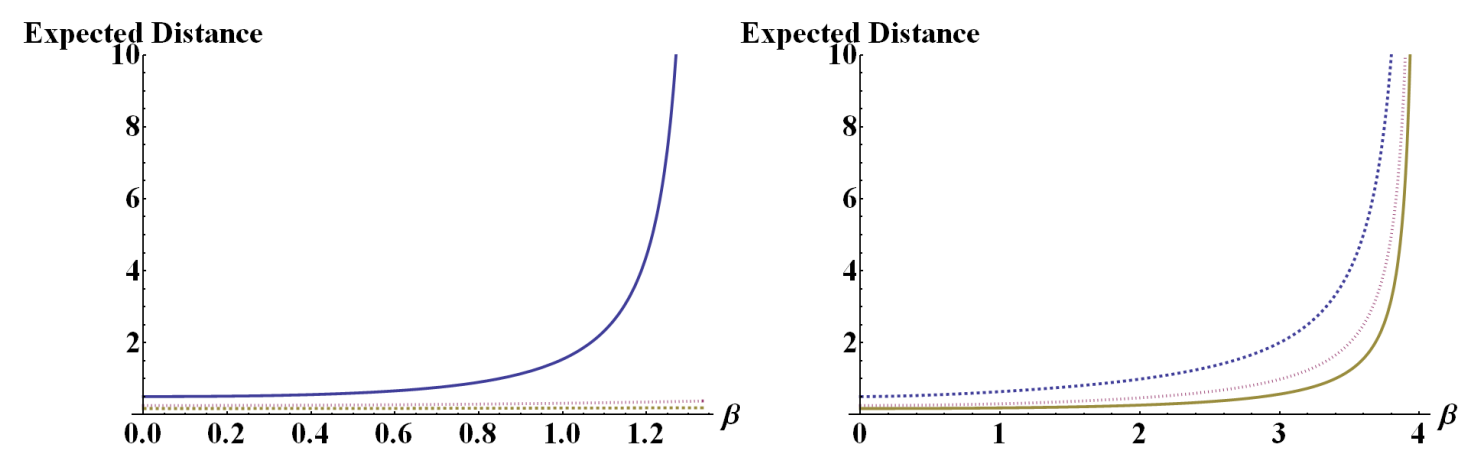

Figure 4: Left panel: Evolution of $\mathcal{P}_{+\beta}(z)$ depending on $\beta$. Right panel: Same for $\mathcal{P}_{-\beta}(z)$.

Plain: Expected distance between the shill and a regular agent, depending on the value of $\beta$. Dotted,Dashed: Expected distance between regular agents, depending on the value of $\beta$.

In the $+\beta$ case, it can be seen that when approaching $\beta_{c}=\frac{4}{3}$, the average distance between the first two agents, which most likely include the shill and a regular fellow, diverges. This divergence reflects the shill's escape from the tight flock formed by the $(N-1)$ regular agents. In the $-\beta$ case, one can observe how the expected distance between the two rear agents also diverges as $\beta \rightarrow g \cdot N=4$. This corresponds to the second critical value for $\beta$. As $\beta$ approaches $g \cdot N$, the common name-based drift of the regular fellows, (i.e $\gamma_{i}=\frac{\beta}{N}$ ), tends to $g$, thus violating the constraint 
Eq.(2). This explains why the distance between the regular agents also diverges in this limiting regime. The extra $\beta$-drift due to the shill is large enough to break global tightness of the flock.

\section{Using a shill to guide the collective crossing of obstacles}

The shill $Y_{1}(t)$ of section (3) has so far been viewed as a mere trouble maker. However, for specific tasks to be achieved, a shill's presence might become truly beneficial. Indeed, the presence of a shill can softly control a swarm towards a preassigned target.

To concretely illustrate this alternative view point, let us, once again, consider a collection of $(N-1)$ identical drifted Brownian (regular) agents and one shill, say $Y_{1}(t)$, all diffusing on $\mathbb{R}$. From now on, we shall represent the evolution on $\mathbb{R}^{2}$ with the time being identified with the $x$-axis, and positions with the $y$-axis. At time $t=0$, all $N$ agents start at location $y_{i}(0)=0,1 \leq i \leq N$. We may think of agents running on the $x$-axis with constant unit speed, as we therefore identify the $x$-axis with time. At the $x$-location $T$, (see Figure 5) we introduce a solid wall (i.e. the obstacle) in which two-slots are drilled. The global objective for the $N$ agents is to try to avoid the fatal collisions with the wall by traveling trough one of the couple of slots. The two slots have width $W$ and are symmetrically drilled at the ordinates $\pm D$, see Figure (5) for a sketch of the configuration.

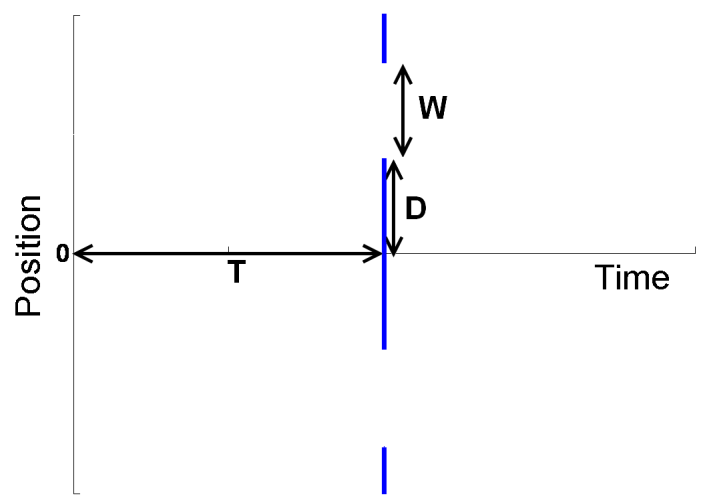

Figure 5: Initial configuration for the soft control problem. The agents start their diffusion at $\left(t_{0}, y_{0}\right)=(0,0)$.

In absence of shill, one has an homogeneous swarm, (all agents are dynamically identical) and the $N$-swarm proceeds according to the rule defined in Eq.(1). As a consequence, the wall will be hit with high probability as, at time $T$, the probability density of the positions will be given by a collection of $N$ centered Gaussians, (we basically have $N$ Brownian motions with constant drifts having their mean barycenter located at zero). As seen in section (3), the presence of a super-diffusive shill $Y_{1}(t)$ may strongly modify this nominal $\beta=0$ picture. Indeed, $Y_{1}(t)$ with suitable parameters $\beta$ and $g$, is able to steer the whole swarm with high preference to one 
of the slots. This basic and simple idea can be made fully quantitative as we now discuss. In our simulations, we set $\beta=0$ once the slots are crossed. Therefore, the global swarm continues its nominal path with a tight configuration after the obstacle.

\subsection{Optimal barycentric driving for suitable choice of $\beta$}

First, we select the $Y_{1}(t)$ parameters to ensure global tightness. This is achieved by defining $\beta<\beta_{c}=\frac{N}{N-1} g$. For a single realisation of the $Z(t)$ noise, we already know that the average barycentric $y$-speed of the swarm is $\frac{ \pm \beta}{N}$. Hence, at time $T$, the group barycenter reaches the $y$-position located at $\frac{ \pm \beta T}{N}$. The center of the slots being located at $\pm\left(D+\frac{W}{2}\right)$, we therefore naturally require:

$$
\frac{ \pm \beta T}{N}= \pm\left(D+\frac{W}{2}\right) \quad \Leftrightarrow \quad \beta_{o p t}:=\frac{\left(D+\frac{W}{2}\right) \cdot N}{T} .
$$

The choice $\beta_{\text {opt }}$, does not yet ensure that the shill $Y_{1}(t)$ itself remains attached to the flock. This second requirement can be achieved provided one has:

$$
\frac{\left(D+\frac{W}{2}\right) \cdot N}{T}=\beta_{o p t}<\beta_{c}=\frac{N}{N-1} g \quad \Leftrightarrow \quad g>\frac{\left(D+\frac{W}{2}\right) \cdot(N-1)}{T}=: g_{c} .
$$

This choice of the couple parameters $\beta_{\text {opt }}$ and $g>g_{c}$ now jointly ensures that i) the barycenter is steered towards one of the slots centers and ii) $Y_{1}(t)$ remains tightly attached to the swarm. The overall swarm's $y$-dispersion, is itself dependent on the diffusion constants $\sigma_{i}$, which were here taken as $\left(\sigma_{i}:=\sigma \forall i\right)$.

For $T=50$, and the choices $\beta=\beta_{\text {opt }}$ and $g=\left(g_{c}+0.1\right) \cdot \sigma$, as $\sigma_{i}=\sigma=1$ for all agents, Figure (6) shows the resulting behaviour for different slot configurations.

\subsection{Adjusting the spatial dispersion resolution for a given drift $g$}

When the drift $g$ is fixed, the ballistic component $\beta$ cannot always be chosen to simultaneously ensure tightness and the collective drive into one of the slots. Keeping the barycentric driving $\beta=\beta_{\text {opt }}$, as defined in Eq.(15), three different scenarii are now possible:

a) $\beta=\beta_{o p t}<\beta_{c}$. In this case, the shill nicely steers the group towards one of the slots while staying attached to the flock, as we already established in section 4.1.

b) $\beta=\beta_{\text {opt }} \geq \beta_{c}$. Here, $Y_{1}(t)$ escapes from the $(N-1)$-flock with a constant drifting velocity. Two sub-cases have to be distinguished, i) drift $\beta-g$ resulting when $+\beta$ is realised by $d Z(t)$ and conversely ii) drift $(N-1) g-\beta$ for the alternative $-\beta$ case. In both cases, the regular agents evolve with an average drift $\gamma_{\text {ave }}$ which reads: 

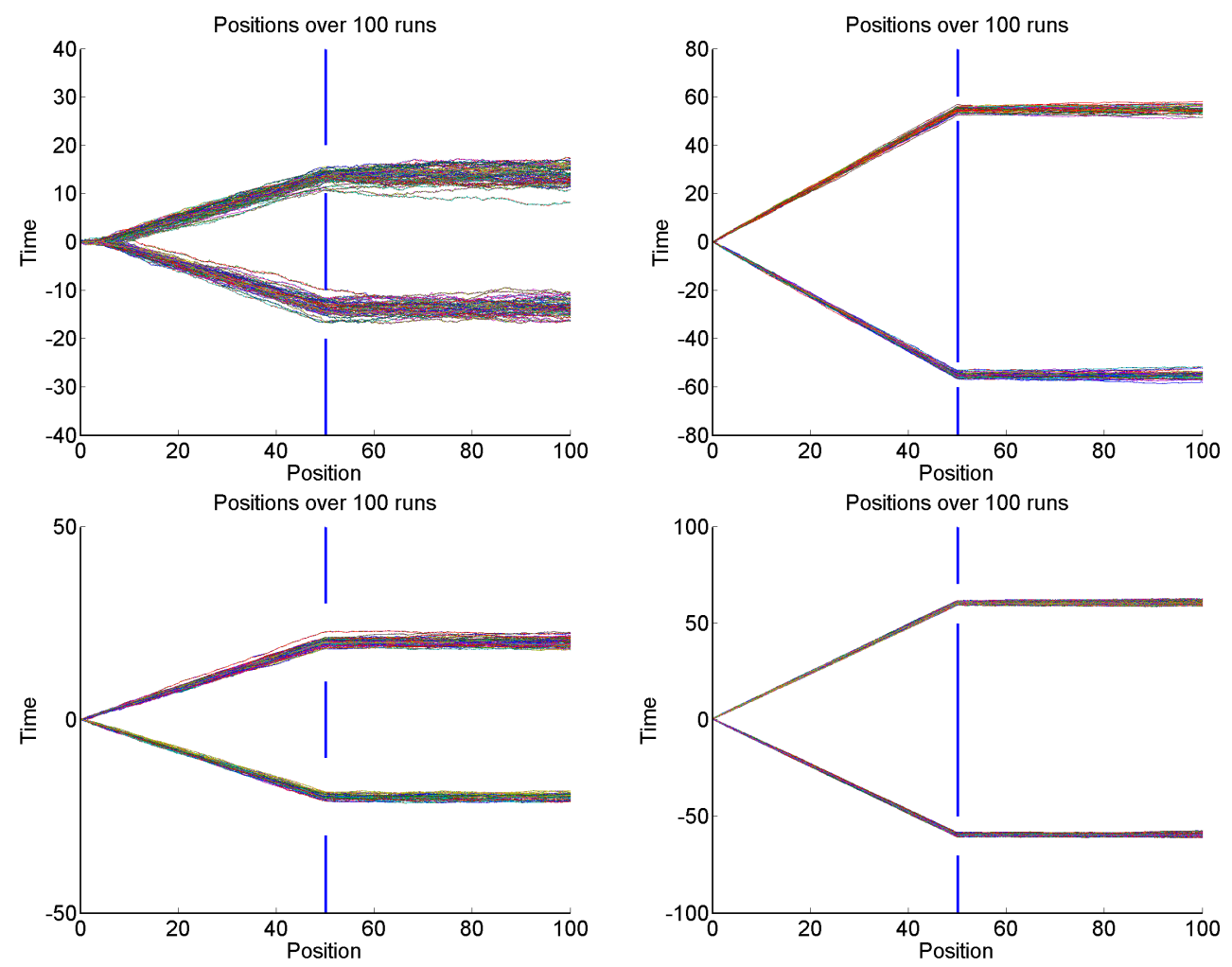

Figure 6: First line: Left: $N=3$ agents, $W=10$ and $D=10$. Right: $N=5$ agents, $W=10$ and $D=50$.

Second line: Left panel: $N=10$ agents, $W=20$ and $D=10$. Right panel: $N=20$ agents, $W=20$ and $D=50$.

$\gamma=\left\{\begin{array}{cll}\frac{\beta}{N}=\frac{(\beta-g) \cdot 1+(N-1) \cdot \gamma_{a v e}}{N} & \text { for }+\beta \text { drift } & \Leftrightarrow \gamma_{a v e}=\frac{g}{N-1} \\ -\frac{\beta}{N}=\frac{((N-1) g-\beta)+(N-1) \gamma_{a v e}}{N} & \text { for }-\beta \text { drift } & \Leftrightarrow \gamma_{a v e}=-g\end{array}\right.$.

c) $\beta=\beta_{\text {opt }} \geq \beta_{c}<N \cdot g$. Here, the shill remains attached to the flock for the $-\beta$ realisation of $d Z(t)$, but escapes otherwise. This then leads to a mix of cases a) and b) depending on whether $+\beta$ or $-\beta$ is realised.

The computation of $\gamma_{a v e}$ from case b) shows that with $g$ fixed such that $\beta_{\text {opt }} \geq \beta_{c}$, the shill escapes from the flock, (hence, no stationary probability measure exists). The remaining $(N-1)$ regular fellows evolve with average speed $\gamma_{\text {ave }}$ (which is $\beta$ independent). Whatever the values taken by $\beta$, the shill is never able to drive the swarm through one of the slots, the swarm's speed being only g-dependent if the shill is not attached to the swarm.

For 100 experiments performed with $N=10, D=20, W=10, T=50$, and a selection of $g$ 's, Figure (7) shows the trajectories of the agents The control parameters used here lead to $g_{c}=4.5$.

The top left panel of Figure (7) shows an experiment where $g<g_{c}$, and $\beta_{\text {opt }}>N \cdot g$. In this case, the shill escapes from the flock in both $\pm \beta$ realisations, and as $g$ is too 

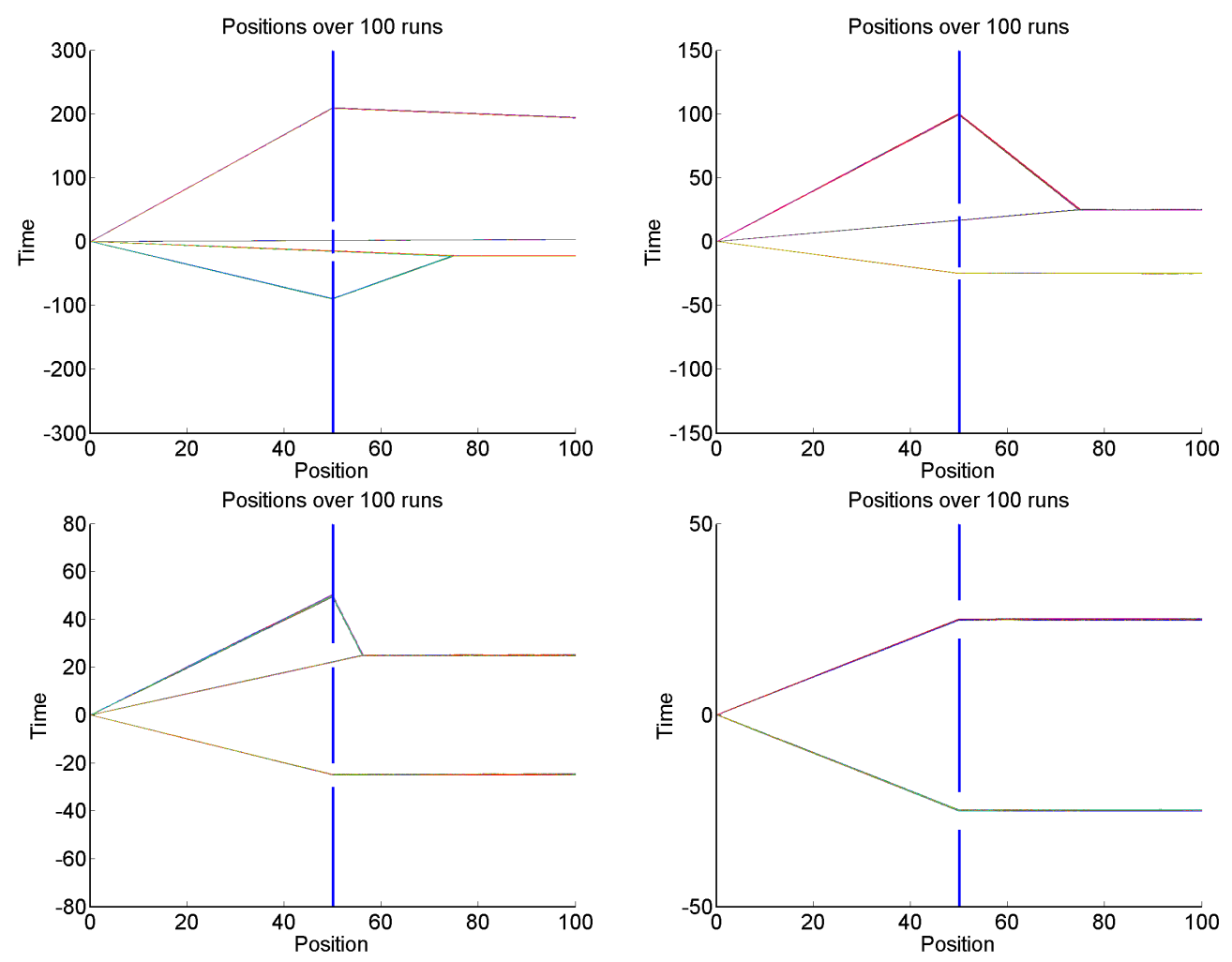

Figure 7: Top line: Left: $g=0.3<g_{\text {opt }}$. Right: $g=3<g_{\text {opt }}$.

Bottom Line: Left: $g=4<g_{\text {opt }}$. Right $g=4.6>g_{\text {opt }}$.

small, the flock ends up in the wall (case b)). In the top right panel and bottom left panels of Figure (7), $g$ is too low to allow the shill to remain attached to the flock for the $+\beta$ realisation (case $c)$ ). But $g$ is different in these cases, changing the average $\mathrm{y}$-speed of the flock $\gamma_{\text {ave }}$. For the $+\beta$ realisation of $d Z(t)$, this lets the swarm hit the wall in the top right panel, but cross safely in the bottom left panel. Finally, in the bottom right panel of Figure (7), $g=4.6$ is large enough to let the shill nicely steer the swarm towards the slots in both $\pm \beta$ realisations, and remains itself attached to the flock (case a)).

So far, only the directions of the swarm has been considered. Obviously, the swarm dispersion is also a determinant feature for efficient slots crossings. Here, not only $g$ but the ratio $\frac{g}{\sigma}$ is determinant. Clearly for small values of $g$, it is likely that the swarm dispersion exceeds the slots widths thus altering the overall efficiency.

\subsection{Soft control for multi-slots configurations}

So far, we did consider the capability of a shill to steer the swarm through a couple of slots. For multi-slots configurations, the shill construction used before naturally suggests to define more general shills to steer swarms in many different directions. This is achievable by replacing the ballistic noise driving the shill with more complex stochastic processes. Doing so however, the shill dynamics cannot be anymore represented by a simple diffusive stochastic differential equation like Eq.(6). As an illustration, consider a three symmetrical slots configuration for which one is 
naturally driven to introduce the following noise source:

$$
\begin{aligned}
& d Z(t)=\left\{\begin{array}{cc}
\beta \tanh (\beta Z(t)) d t+d W(t) & \text { with prob. } \frac{2}{3} \\
d W(t) & \text { with prob. } \frac{1}{3}
\end{array}=\right. \\
& =\left\{\begin{array}{c}
\text { ballistic noise with drift }+\beta \text { with prob. } \frac{1}{3} \\
\text { white gaussian noise with prob. } \frac{1}{3} \\
\text { ballistic noise with drift }-\beta \text { with prob. } \frac{1}{3}
\end{array} .\right.
\end{aligned}
$$

The shill dynamics is taken as before, namely with probability $\frac{2}{3}$ it steers the swarm towards the positive or negative slots with ballistic parameter $\beta$ and with probability $\frac{1}{3}$, it behaves as a regular agent driving the swarm on centered path, see Figure (8).
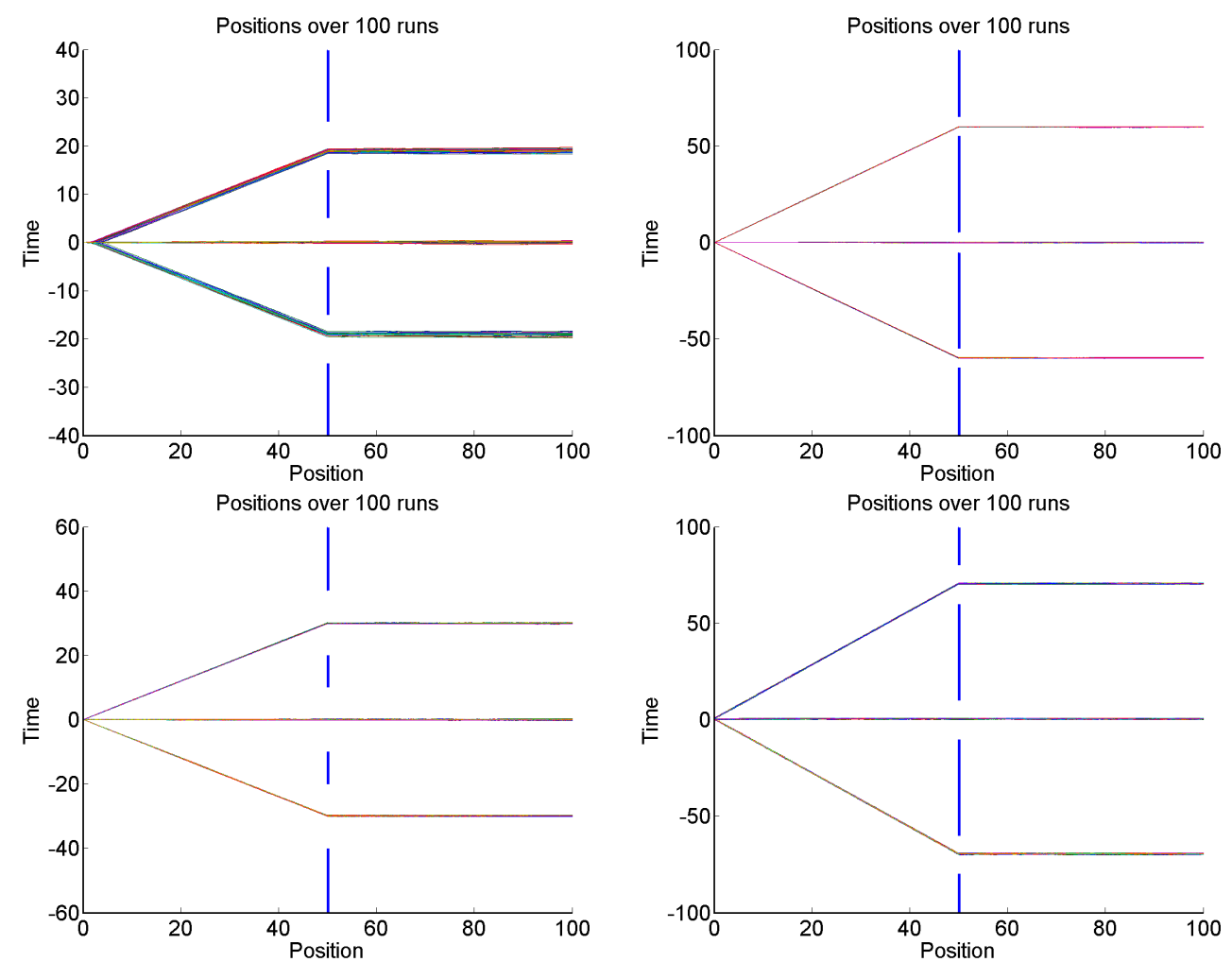

Figure 8: Trajectories for $\beta$ and $g$ optimal, with $N_{d}=3$ doors, under different conditions for the position/width of the doors.

For arbitrary number of slots and configurations, one can generalize our construction by suitably adjusting the properties of the noise source driving the shill.

\section{Summary and conclusions}

In societies of interacting autonomous agents, it commonly occurs that each individual dynamics differ, leading therefore to truly heterogeneous collections of agents. The characterization of the spatio-temporal patterns that can emerge from 
such heterogeneous societies offers a true challenge from both experimental and analytical research. Indeed, for heterogeneous fellows, analysis is usually made complex as the use of ordinary statistical mechanics tools, like the mean-field approach, are ruled out. The present paper, based on the dynamics of ranked-based Brownian motions, unveils a class of analytically soluble models involving an arbitrary number of heterogeneous interacting agents. Heterogeneity opens a wide range of possible interpretations and makes models suitable for numerous interdisciplinary implementations. Here, we focus on the simplest combinatoric situation where a single agent, the shill, infiltrates a community of homogeneous fellows. By mutual interactions with the shill, new dynamical patterns for the whole population emerge and can be analytically discussed. As in all actual situations, the shill's intervention can be either perceived as destructive or constructive. As an illustration, a tight population of ranked-based interacting Brownian motions can be destroyed by the very presence of a "turbulent" super-diffusive shill and this cohesion lost can be considered as negative. Concurrently, the same shill's action may also, in certain circumstances, be counted as beneficial. As we show analytically, a single shill can alone induce an average trajectory deflection of the whole swarm. This effective soft control might positively contribute to help the global population to avoid the destructive effects of collisions with an obstacle. We barely scratch the whole wealth of analytical possibilities offered by such stylized models. We believe that this class of dynamics provides a truly relevant tool to investigate the cooperative spatio-temporal patterns emerging from interacting heterogeneous agents.

\section{References}

[1] R. Fernholz A. D. Banner and I. Karatzas. Atlas models of equity markets. Ann. Appl. Probab. 15, 2296-2330, 2005 .

[2] J.-L. Deneubourg H. Bleuler A. Gribovskiy, J. Halloy and F. Mondada. Towards mixed societies of chickens and robots. IEEE/RSJ 2010 International Conference on Intelligent Robots and Systems, Conference Proceedings pp. 4722-4728, 2010.

[3] C. Escudero I. A. Couzin J. Buhl I. G. Kevrekidis P. K. Maini C. A. Yates, R. Erban and D. J. T. Sumpter. Inherent noise can facilitate coherence in collective swarm motion. PNAS 14, vol. 106, 5464-5469, 2009.

[4] S. Chatterjee and S. Pal. A phase transition behaviour for brownian motions interacting through their ranks. Probab. Theory Relat. Fields 147, 123-159, 2010.

[5] V. Dosseti. Cohesive motion in one-dimensionnal flocking. Physica A: Math. Theor. 45, 2012.

[6] J. Han and L. Wang. New strategy of the shill: Consistent moving. Proceedings of the 29th Chinese Control Conference, pp. 4459-4464, 2010.

[7] R. O. Clement I. D. Couzin N. Holt A. J. W. Ward D. Waters J.J. Faria, J. R. G. Dyer and J. Krause. A novel method for investigating the collective behaviour of fish: Introducing 'robofish'. Behavioral Ecology and Sociobiology 8, vol. 64, 1211-1218, 2010.

[8] Ph. Blanchard M.-O. Hongler, R. Filliger and J. Rodriguez. "Contemporary Topics in Mathematics and Statistics with Applications" in "On Stochastic Processes Driven By Ballistic Noise Sources". Ed. M. R. Adhikari, 2012.

[9] R. Filliger M.-O. Hongler and Ph. Blanchard. Soluble models for dynamics driven by a super-diffusive noise. Physica A 370, 301-355, 2006.

[10] S. Pal and J. Pitman. One dimensional brownian particles with rank-dependent drifts. Ann. Appl. Probab. 18 2179-2207, 2008

[11] J. Henderson A. Frost R. Vaughan, N. Sumpter and S. Cameron. Experiments in automatic flock control. Robotics and Autonomous Systems 1, vol. 31, 109-11\%, 2000. 
[12] L.C.G Rogers and J.W. Pitman. Markov functions. Ann. of Prob. 9(4), 573-582, 1981.

[13] A. Banner I. Karatzas T. Ichiba, V. Papathanakos and R. Fernhold. Hybrid atlas model. Ann. Appl. Probab. 21(2) 609-644, 2011.

[14] I. Giardina T. Mora E. Silvestri M. Viale W. Bialek, A. Cavagna and A. M. Walczak. Statistical mechanics for natural flocks of birds. PNAS 13, vol. 109, 4786-4791, 2012.

[15] L. Wang and L. Guo. Robust consensus and soft control of multi-agent systems with noises. Journal of Systems Science and Complexity 3, vol. 21, 406-415, 2008.

[16] J. Han X. Wang and H. Han. Special agents can promote cooperation in the population. PLoS ONE 12, vol. $6,2011$.

[17] X.-Z. Mao Y. Wang and J.-P. Liu. Soft control for swarm systems with simple attraction and repulsion functions. Proceedings of the 2nd International Conference on Intelligent Networks and Intelligent Systems, pp. 482-485, 2009. 